\title{
Glycoproteomics revealed novel N-glycosylation biomarkers for early diagnosis of lung adenocarcinoma cancers
}

\section{Kai Fang}

The second Affiliated Hospital of Chengdu Medical College

\section{Qin Long}

The second Affiliated Hospital of Chengdu Medical College

Zhonghua Liao

The second Affiliated Hospital of Chengdu Medical College

Chaoyu Zhang

The second Affiliated Hospital of Chengdu Medical College

\section{Zhiqiang Jiang ( $\boldsymbol{O}$ jiangzq_416@163.com )}

The second Affiliated Hospital of Chengdu Medical College

\section{Research Article}

Keywords: lung adenocarcinoma, early diagnosis, circulating biomarkers, $\mathrm{N}$-glycosylation, machine learning

Posted Date: January 18th, 2022

DOI: https://doi.org/10.21203/rs.3.rs-1256117/v1

License: (c) (i) This work is licensed under a Creative Commons Attribution 4.0 International License. Read Full License 


\section{Abstract}

Circulating biomarkers play important roles in diagnosis of malignant tumors. N-glycosylation is an important post-translation patter and obviously affect biological behaviors of malignant tumor cells. However, the role of $\mathrm{N}$-glycosylation sites in early diagnosis of tumors still remains further investigation. In this study, plasma from 20 lung adenocarcinoma (LUAD), which were all classified as stage I, as well as 20 normal controls (NL) were labeled and screened by mass spectrometry (MS). Total 39 differential $\mathrm{N}$-glycosylation sites were detected in LUAD, 17 were up-regulated and 22 were down-regulated. In all differential sites, ITGB3-680 showed highest potential in LUAD which showed 99.2\% AUC, $95.0 \%$ SP and 95.0\% SN. Besides, APOB-1523 (AUC: 89.0\%, SP: 95.0\%, SN: 70.0\%), APOB-2982 (AUC: 86.8\%, SP: 95.0\%, SN: 45.0\%) and LPAL2-101 (AUC: 81.1\%, SP: 95.0\%, SN: 47.4\%) also acted as candidate biomarkers in LUAD. Combination analysis was then performed by random forest model, all samples were divided into training group (16 cases) and testing group (4 cases) and conducted by feature selection, machine learning, integrated model of classifier and model evaluation. And the results indicated that combination of differential sites could reach $100 \%$ AUC in both training and testing group. Taken together, our study revealed multiple N-glycosylation sites which could be applied as candidate biomarkers for early diagnosis diagnosis of LUAD.

\section{Introduction}

Lung cancer (LC) is the leading cause of malignant cancer-related mortality worldwide. Lung cancer can be divided into two histological subtypes, non-small cell lung cancer (NSCLC) and small cell lung cancer (SCLC), and NSCLC accounts for $85 \%$ of all lung cancer cases [1]. Lung adenocarcinoma (LUAD) (50\%), squamous carcinoma (LUSC) (35\%) and large cell carcinoma (15\%) are the three major subtypes of NSCLC [2]. Despite advanced therapeutic strategies, including target therapy and radiation therapy, the 5year survival rate of lung cancer still remain in less than $20 \%$ [3]. The reason of high mortality of lung cancer dues to late diagnosis and early diagnosis will contribute greatly to better prognosis of LC patients [4]. Low-dose computed tomography (CT) has been widely applied clinically and increased early diagnosis of LC by detecting small malignant nodule [5]. However, radiation injury, high financial burden, especially high-false positive rate are points of controversy [6]. Circulating biomarkers, including proteins, exosomes, miRNA and circulating free DNA (cfDNA), play important roles in detecting primary lung cancer due to features of noninvasive, convenient and inexpensive acquisitions [7]. Classical tumor biomarkers include carcinoembryonic antigen (CEA) [8], carbohydrate antigen 19-9 (CA199) [9], carbohydrate antigen 12-5 (CA125) [10], as well as LUSC specific marker Cyfra21-1 [11] and SCLC specific marker (NSE) [12]. But most of these markers is only sensitive in advanced (stage III+IV) patients and contribute poorly to early diagnosis of lung cancer. Therefore, it is very important to identify novel biomarkers which can be applied for early screening and diagnosis of lung cancer.

Recently, more studies report novel types of circulating molecules which act as candidate biomarkers in early diagnosis of lung cancer, including circulating tumor DNA (ctDNA) [13], exosomes [14], circulating tumor cells (CTC) [15] and autoantibodies [16]. Exosomes are lipid and nanosized (30-150nm diameter) 
vesicles which are secreted by almost all cell types and released into extracellular microenvironment [17]. Exosomes can participate in tumor growth and metastases by exchanging oncogenic molecules with nearby and distant cells to establish suitable microenvironment [18]. Exosomes contain multiple contents, such as proteins, lipids, nucleic acid and metabolites, which can be applied as biomarkers for tumor diagnosis [19]. Autoantibodies (AAbs) are produced by immune response against specific tumor antigens formed by mutated genes and aberrantly expressed proteins, which are currently an option of tumor immune-related markers for malignant tumors [20]. Although rapid degradation and clearance in serum levels limits their clinical application, AAbs appear to be a promising method for in distinguishing normal individuals and non-malignant benign disease.

Besides above tumor-related molecules, glycoproteomic technologies, based on mass spectrometry, has now been applied in discovery of biomarker for diagnosis of malignant tumors [21]. In this study, we conducted glycoproteomic assay for 20 LUAD patients, which were all classified as stage I and 20 healthy controls $(\mathrm{NL})$ to screen novel glycoprotein biomarkers for early diagnosis of LAUD.

\section{Materials And Methods}

\section{Participants and samples}

This study enrolled two groups, LUAD patients were from the Department of Respiration and Thoracic Surgery, and all samples were obtained from patients who had not obtained surgery or other treatment such as chemical, target or radiation therapy. All clinical characteristics, including age, gender and TNM stage were accessed from medical records. All healthy controls were from the Center of Health Management, participants were exclude if their family had any history of cancer. Plasma samples were extracted and stored in $-80^{\circ} \mathrm{C}$ before applied for detection. The utilization of human samples was approved by Medical Ethics Committee and Institutional review board of China National Nuclear Corporation 416 Hospital, with all participants providing written informed consents. Plasma samples were extracted and stored in $-80^{\circ} \mathrm{C}$ according to standard procedures.

\section{Protein extraction and TMT labeling}

All plasma samples were centrifugated at $12000 \mathrm{~g}$ at $4^{\circ} \mathrm{C}$ for $10 \mathrm{~min}$ to remove cellular debris. The supernatant was then transferred to a new $1.5 \mathrm{ml}$ tube and BCA kit was introduced to detect protein concentration in accordance to the manufacturer's manual. Then, all plasma samples were treated by $5 \mathrm{mM}$ dithiothreitol at $56^{\circ} \mathrm{C}$ for $30 \mathrm{~min}$ to digest protein solution and alkylated with $11 \mathrm{mM}$ iodoacetamide for $30 \mathrm{~min}$ at room temperature in darkness. All samples were diluted by adding 100mM TEAB to urea concentration less than $2 \mathrm{M}$. For protein digestion, trypsin was added at 1:50 mass ration (trypsin-toprotein) overnight for first digestion and 1:100 ratio mass for second digestion (4 hours).

All digested peptides were desalted by Strata XC18 SPE column (Phenomenex) and vaccum-dired. Peptides were then reconstituted in 0.5M TEAB and processed by TMT kit/iTRAQ kit according to manual 
instruction. The peptide mixtures were incubated at room temperature for 2 hours and pooled, desalted and dried by vacuum centrifugation.

\section{HPLC fractionation, affinity enrichment and LC-MS/MS analysis}

High pH reverse-phase HPLC (Thermo Betasil C18 column) was performed to fractionate tryptic peptides (5um particles, $10 \mathrm{~mm} \mathrm{ID,} 250 \mathrm{~mm}$ length). All peptides were separated with a gradient of $8 \%$ to $32 \%$ acetonitrile ( $\mathrm{pH} 9.0$ ) into 60 fractions more than 1 hour. And the peptides were combined into 4 fractions and dried by vacuum centrifuging.

Tryptic peptides were incubated with pre-washed antibody beads (Lot number 2326391910488716510, PTM Bio) at $4^{\circ} \mathrm{C}$ overnight with gentle shaking after dissolving in NETN buffer ( $100 \mathrm{mM} \mathrm{NaCl}, 1 \mathrm{mM}$ EDTA, $50 \mathrm{mM}$ Tris-HCl, 0.5\% NP-40, pH 8.0) for enrichment. Then NETN buffer were added to wash beads and $0.1 \%$ trifluoroacetic acid were added to elute bound peptides from beads. Finally the eluted fractions were combined and vacuum-dried and the peptides were desalted with C18 ZipTips (Millipore) for LCMS/MS analysis.

The enriched peptides were dissolved in $0.1 \%$ formic acid (solvent $A$ ) and loaded onto a home-made reversed-phase analytical column (15-cm length, $75 \mu \mathrm{m}$ i.d.). The gradient increased from $6 \%$ to $23 \%$ in solvent B ( $0.1 \%$ formic acid in $98 \%$ acetonitrile) over $26 \mathrm{~min}, 23 \%$ to $35 \%$ in $8 \mathrm{~min}$ and reached to $80 \%$ in 3 min then holding at $80 \%$ for the last 3 min, all were treated at a constant flow rate of $400 \mathrm{~nL} / \mathrm{min}$ in EASYnLC 1000 UPLC system. The peptides were subjected to NSI source followed by tandem mass spectrometry (LC-MS/MS) in Q ExactiveTM Plus (Thermo). The electro-voltage was $2.0 \mathrm{kV}$ and the $\mathrm{m} / \mathrm{z}$ scan ranged from 350 to 1800. All intake peptides were selected for LC-MS/MS using NCE setting as 28 and the fragments were detected in the Orbitrap at a resolution of 17,500. A data-dependent procedure was then conducted to peptides and alternated between one MS scan followed by 20 LC-MS/MS scans with 15.0s dynamic exclusion.

\section{Database search}

Maxquant search engine (v.1.5.2.8) was performed to process the LC-MS/MS data. Tandem mass spectra were searched in Human uniprot database together with reverse decoy database. The mass tolerance for precursor ions was set as $20 \mathrm{ppm}$ in first search and $5 \mathrm{ppm}$ in main search, and the mass tolerance for fragment ions was set as $0.02 \mathrm{Da}$. Carbamidomethyl on Cys was specified as fixed modification and acetylation modification and oxidation on Met were specified as variable modifications. FDR was adjusted to $<1 \%$ and minimum score for modified peptides was set $>40$.

\section{GO and domain annotation}

Gene Ontology (GO) annotation proteome was derived from UniProt-GOA database ( http://www.ebi.ac.uk/GOA/). Identified protein ID was converted to UniProt ID and then mapped to GO ID. The InterProScan soft was applied to annotated protein's GO functional based on protein sequence alignment method once the identified proteins were not annotated by UniProt-GOA database. All proteins 
were distinguished by Gene Ontology annotation based on three categories: biological process, cellular component and molecular function.

InterPro domain database was performed to identify proteins domain which were annotated by InterProScan (a sequence analysis application) based on protein sequence alignment method

\section{KEGG Pathway Annotation and Subcellular Localization}

Kyoto Encyclopedia of Genes and Genomes (KEGG) was a networks which connected all known molecular interaction, including pathways and complexes, information about genes and proteins, as well as and information about biochemical compounds and reactions. KEGG pathway mainly contained metabolism, genetic information processing, environmental information processing, cellular processes, rat diseases and drug development. In protein annotation, we firstly used KEGG online service tools KAAs to annotate the protein description in KEGG database. Then KEGG online service tools KEGG mapper was conducted to mapping the annotation results.

Wolfpsort was an updated version of PSORT/PSORT II for the prediction of eukaryotic sequences, which was introduced in this study to predict subcellular localization. Special for protokaryon species, Subcellular localization prediction soft CELLO was used.

\section{Motif analysis}

Soft MoMo (motif-x algorithm) was then applied to identify the model of sequences constituted with amino acids in specific positions of modify-21-mers (10 amino acids upstream and downstream of the site) in all protein sequences. And all the database protein sequences were used as background database parameter. Minimum number of occurrences was set to 20 . Emulate original motif- $x$ was ticked, and other parameters with default.

\section{Functional of Gene Ontology enrichment}

GO annotation was performed to separate proteins into three categories: biological process, cellular compartment and molecular function. For each category, two-tailed Fisher's exact test was used to test the enrichment of the differentially modified protein against all identified proteins and pathway enrichment was identified by two-tailed Fisher's exact test. These pathways were classified into hierarchical categories according to the KEGG website. For each category proteins, InterPro database was studies and two-tailed Fisher's exact test was employed to test the enrichment of the differentially modified protein against all identified proteins. For all analysis, $p$-value $<0.05$ was considered significant.

\section{Enrichment-based Clustering}

To further hierarchical cluster based on differentially modified protein functional classification (such as: GO, Domain, Pathway, Complex), we collected all available categories after enrichment along with $P$ values and filtered for those categories which were at least enriched in one of the clusters with $P$ value 
$<0.05$. This filtered $P$ value matrix was transformed by the function $x=-\log 10$. All $x$ values were $z-$ transformed for each functional category and $z$ scores were clustered by one-way hierarchical clusteringin Genesis and the cluster membership were visualized by "heatmap.2" function from the "gplots" R-package.

\section{Protein-proteins Interaction Network}

To investigate protein-protein interactions, all differentially expressed modified protein database accession or sequence were searched in STRING database (version 10.0). Only interactions between the proteins belonging to the searched data set were selected, STRING defines a metric called "confidence score" to define interaction confidence; we fetched all interactions that had a confidence score $\geq 0.7$ (high confidence). Interaction network form STRING was visualized in R package "networkD3".

\section{Results}

\section{Study design and clinical characterizations of all patients}

Total 40 participants were enrolled in this study, including 20 LUAD patients and 20 healthy controls (NL). The average age was $57.8 \pm 10.8$ and $45.1 \pm 11.4$ in LUAD and NL groups, and LUAD included 6 male and 14 female, while NL contained 7 male and 13 female. All LUAD patients were classified as stage I according to $7^{\text {th }}$ version, and 7 had smoke history while 13 were never smoking. All clinical features of all participants were listed in Table 1.

Table 1. All clinical features of all participants were listed in Table. 


\begin{tabular}{llllll} 
Number & Sex & age & smoke history & TNM & stage \\
\hline LA1 & Female & 75 & No & T1bN0M0 & IA2 \\
\hline LA2 & Male & 61 & Yes & T1bN0M0 & IA2 \\
\hline LA3 & Female & 37 & No & T1aN0M0 & IA1 \\
\hline LA4 & Male & 63 & Yes & T1cN0M0 & IA3 \\
\hline LA5 & Female & 62 & No & T1bN0M0 & IA2 \\
\hline LA6 & Male & 61 & Yes & T1c N0M0 & IA3 \\
\hline LA7 & Female & 57 & No & T2aN0M0 & IB \\
\hline LA8 & Female & 51 & No & T1bN0M0 & IA2 \\
\hline LA9 & Male & 51 & No & T1bN0M0 & IA2 \\
\hline LA10 & Female & 46 & No & T1b/2aN0M0 & IA2/ \\
\hline LA11 & Female & 49 & No & T1cN0M0 & IA3 \\
\hline LA12 & Female & 42 & No & T1bN0M0 & IA2 \\
\hline LA13 & Female & 60 & No & T1aN0M0 & IA1 \\
\hline LA14 & Female & 57 & No & T2aN0M0 & IB \\
\hline LA15 & Male & 70 & Yes & T1bN0M0 & IA2 \\
\hline LA16 & Male & 82 & No & T2aN0M0 & IB \\
\hline LA17 & Female & 65 & No & T1bN0M0 & IA2 \\
\hline LA18 & Female & 59 & No & T1bN0M0 & IA2 \\
\hline LA19 & Female & 63 & No & PT1bN0M0 & IA2 \\
\hline LA20 & Female & 45 & No & IB \\
\hline
\end{tabular}

To explore N-glycoprotein sites for lung cancer diagnosis, plasma were obtained from LUAD and NL controls and were extracted and labelled. After fractionation and enrichment, LC-MS/MS was performed to investigate the $\mathrm{N}$-glycoprotein levels in all samples. The differential $\mathrm{N}$-glycoprotein levels between LUAD and NL were analyzed based on databases. And the candidate $\mathrm{N}$-glycoprotein sites were combined to study the role of novel biomarkers for future lung cancer diagnosis. The study procedures were listed in Figure 1.

\section{Characteristics of the identified N-glycoproteins}

Next, we performed enriched peptides and detected by LC-MS/MS to identify N-glycoproteins in obtained plasma samples. In comparison to NL samples, we obtained total 383,675 spectrums in LUAD patients 
and 18,566 matched spectrums were identified based on protein datasets. We then identified total 4385 peptides in matched spectrums. In all peptides, 1399 were belongs to modified peptides which contained 502 identified sites. In LUAD samples, 478 sites were quantified which were identified from 275 proteins (Figure 2A).

In this study we identified total 478 sites in 263 proteins in LUAD patients. The number of $\mathrm{N}$-glycosylated sites assigned to all proteins ranged from 1 to 11 with average degree of glycosylation was 2.5. More than half of glycoproteins $(270 / 478,57.1 \%)$ carried only a single N-glycoprotein site, $110(23.6 \%)$ of them harbored double N-glycoprotein sites (Figure 2B, 2C). Triple and four N-glycoprotein sites were 41 (9.1\%) and $27(6.2 \%)$, respectively. And the rest $17(4 \%)$ contained five $(8,2.5 \%)$, eight $(1,0.4 \%)$, nine $(3,0.7 \%)$ and eleven $(1,0.4 \%)$ sites (Figure 2B, 2C). And our results also indicated the overlap numbers in all samples in both protein and N-glycoprotein sites (Figure 2D, 2E). In summary, multiple proteins and Nglycosylation sites were identified in LUAD patients, which could be conducted to further analysis to identify candidate biomarkers for future clinical application.

\section{Disease-associated changes in N-glycopeptide abundance in LUAD}

In comparison to NL samples, 39 differential N-glycosylation sites were obtained in LUAD. In all differential sites, 17 increased in LUAD patients, such as APOB-2982, SERPINC1-224 and APOB-1523, while 22 decreased in lung cancer samples, including ITGB3-125 and VWF-235 (Figure 3A). We analyzed the cellular distribution of the differential proteins, and the result indicated 24 proteins were extracellular, 3 proteins were endoplasmic reticulum, while the cytoplasm, cytoskeletio, mitochondria and plasma membrane contained 1 protein (Figure 3B).

The neighborhood residues of glycosylated asparagines could determine the specificity of LUAD. MoMo was conducted to obtain the characteristic sequence of modified sites and their enrichment statistics. As shown in Figure 3C, 2 conserved amino acids flanking the glycosylated asparagine residues (from - 10 to +10) were defined. These motifs included $N-x-T-*-Y$ and $N-x-S$, where $x$ represented any amino acid except proline and the asterisk denoted a random amino acid. Based on analysis of hierarchical clusters, threonine and serine displayed the highest probability at the position +2 , while the frequency of a proline residue in the proximity was markedly underrepresented (Figure 3D). Taken together, our results suggested a preference motif exposed to the surface of glycoproteins.

\section{Analysis and annotation of differentially N-glycosylated proteins in LUAD}

To elucidate the potential functions of those quantifiable proteins in LUAD samples, we analyzed the quantifiable proteome data set for three enrichment gene ontology (GO) categories: molecular function, cellular compartment and biological process. Based on accumulative normal distribution, we divided all pathways into 4 quantiles: Q1 (<0.769 fold change), Q2 (0.769-0.833 fold change), Q3 (1.2-1.3 fold change) and Q4 (>1.3 fold change). In the biological process category, the significant increased pathways enriched in cell migration process such as tissue remodeling, cell growth, cell-matrix adhesion and actin cytoskeleton organization (Q1), and also contained immune-related regulation including leuckocyte 
chemotaxis, lymphocyte migration, cytokine stimulus and T cell migration (Q2). In Q3 and Q4 analysis, we also found that activated pathways in LUAD samples aggregated in innate immune response, endocytosis (Q3) and in metabolic transport process including protein, lipid, sterol and cholesterol (Q4) (Figure 4A). As to cellular component analysis, the results revealed that assembled components in LUAD patients were intrinsic, integral, vesicle, cytoplasmic and organelle membrane (Q1), trans-Golgi network, vesicle transport and secretory granule lumen (Q2), metabolic components (protein-lipid complex, plasma lipoprotein particle and lipoprotein particles) (Q3) and endocytic vesicles (Q4) (Figure 4B). Finally we analyzed molecular function pathways in LUAD samples, we observed enrichment focused on binding activity, including protease, fibronectin, carbohydrate and sulfur compound (Q1), enzyme, cytokine and growth factor (Q2), as well as transport activity (Q3) and lipoprotein receptor binding (Q4) (Figure 4C). We then analyzed KEGG pathways in LUAD samples and found that the enrichment contained neutrophil extracellular formation, platelet activation (Q1) and amoebiasis (Q2) (Figure 4D).

Finally STRING protein-protein interaction (PPI) dataset was performed to analyze the protein-protein interactions in identified proteins. The results indicated that APOB, SERPINC1 and CLU were the core nodes in interaction network, which interacted with multiple proteins such as IGF2R, C8A, HPX, HRG and ADAM10. FN1 and SERPING1 also acted as central connection nodes, which interacted with SERPINA5, ITGB3, VWF and F5 (Figure 4E). Taken together, our results indicated that by TMT labeling and LCMS/MS sequencing, we identified multiple differential proteins and $\mathrm{N}$-glycosylation sites in LUAD patients, which harbored multiple pathways in tumor-related abnormal metabolism and protein transport.

\section{The role of $\mathrm{N}$-glycosylation sites in diagnosis of LUAD}

To further explore the diagnostic accuracy of candidate biomarkers in LUAD, we performed ROC analysis to define the sensitivity (SN) and specificity (SP) of identified N-glycosylation sites. The results suggested that multiple $\mathrm{N}$-glycosylation sites in proteins harbored valuable roles in lung cancer diagnosis. The tope 4 proteins were ITGB3-680, APOB-1523, APOB-2982 and LPAL2-101, which all showed AUC (area under curve) $>80.0 \%$ (Figure 5A). The most important site was ITGB3-680, the AUC was $99.2 \%$, SN (sensitivity) and SP (specificity) were both $95.0 \%$ in compared with NL group. In APOB-1523 analysis, the AUC was $89.0 \%$, SN and SP were $70.0 \%$ and 95/0\%, respectively. In APOB-2982 analysis, AUC was $86.8 \%$, SN was 45.0\% and SP was $95.0 \%$ when compared with NL group. The AUC in LPAL2-101 analysis was $81.1 \%$, while $\mathrm{SN}$ was $47.4 \%$, SP was $95.0 \%$ (Figure $5 \mathrm{~A}$ ).

Besides top 4 proteins, we also analyzed other candidate proteins. We found that AUC of CLU-291 was $78.5 \%$, SN was $50.0 \%$ and SP was $95.0 \%$. And the AUC, SN, SP of VWF-2357 were $76.8 \%, 50.0 \%$ and $95.0 \%$, respectively. In C8A-437 analysis, the AUC was $76.5 \%$, SN was $45.0 \%$, SP was $95.0 \%$. In CD109-247 analysis, we obtained AUC was $76.5 \%$, SN was $25.0 \%$ and SP was $95.0 \%$ (Figure $5 B$ ).

Finally we investigate ECM1-444, CFH-882, VNN1-283 and AFM-33. The analysis results indicated that in ECM1-444, the AUC was 75.6\%, SN was $30.0 \%$ and SP was $95.0 \%$. In CFH-882, the AUC was $75.5 \%$, SN was $25.0 \%$, SP was $95.0 \%$. In VNN1-283 analysis, the AUC was $75.5 \%$, SN was $60.0 \%$ and SP was $90.0 \%$. 
In AFM-33 analysis, the result showed that AUC was $75.3 \%$, while SN and SP were $60.0 \%$ and $95.0 \%$, respectively (Figure 5C).

In summary, our study revealed multiple $\mathrm{N}$-glycosylation sites harbored highly potential diagnostic value in LUAD diagnosis.

\section{Combination analysis of novel N-glycosylation sites by machine learning model}

In this part, we introduced machine learning model to test the diagnosis efficiency of candidate biomarkers in lung cancer. We divided all participants into training set (establish model and adjust parameters, 16 cases) and test set (evaluate the model, 4 cases). By combining feature selection, machine learning algorithm, classifier integration method and dataset validation, random forest model was conducted to determine whether the proteomic profile had cancer-specific features for lung cancer diagnosis (Figure 6A). Due to small sample sizes of two data sets, 2/3 individuals in the training set were selected to grow decision trees by boostrapping and the remaining participants were used as out of bag samples for cross-validation importance. In feature selection, each sample was represented by feature vector, which contained 24 expression features and each expression feature has different ability to distinguish different types of samples. Univariate feature analysis was introduced to quantify the ability of expression features in distinguishing different samples and we could calculate the correlation between each feature and sample types by variance test. Based on this method, the feature scores and $p$ value of candidate molecules revealed the top 15 molecules in candidate N-glycosylation sites, including ITGB3680, APOB-2982, CLU-291, ECM1-444, C8A-437, VNN1-283, LPAL2-101, APOB-1523, BTD-56, AFM-33, APOB-3411, AFM-402, CFH-882, CRISP3-270, SERPINA5-262, IGHG4-177 and SERPING1-238, which were performed for model construction (Figure 6B). Next, we analyzed the Pearson correlation coefficient to understand the linear correlation of top 5 proteins (ITGB3-680, APOB-2982, CLU-291, ECM1-444 and C8A437), the results indicated that ITGB3-680, ECM1-444 and C8A-437 correlated closely (0.459 and 0.368) while APOB-2982 and CLU-291 had correlation (0.357) (Figure 6C). Finally machine learning model was established to evaluate the role of candidate biomarkers in lung cancer diagnosis. In this study we used logistic regression, support vector machine and random forest as base classifier to construct voting classifier. To evaluate the differences between prediction and actual category, four calculation accuracy index, including sensitivity, specificity, Mattthews' correlation coefficient and AUC (area under curve) were introduced in this model. In all expression features, the optimal expression feature subset in current data set was selected to obtain optimal prediction accuracy by incremental feature selection (IFS). The AUC curves in training and test set were obtained by plotting the true positive rate against the false positive rate under different cut-off values, and the result indicated that AUC reached $100 \%$ in both training and test sets (Figure 6D). In summary, the machine learning model revealed that combination of $\mathrm{N}$ glycosylation sites had important application in lung cancer diagnosis.

\section{Discussion}


Majority lung cancer patients are diagnosed at advanced stages due to lack of effectively early diagnostic strategies. Thus, it is important to identify more valuable biomarkers for early diagnosis of lung cancer. Nowadays, many novel circulating biomarkers had valuable potential in lung cancer diagnosis, including circulating tumor DNA (ctDNA), circulating tumor cells, auto-antibodies and exosomes. N-linked glycosylation is one of the most important post-translational modifications in malignant tumors, which were important in numerous regulations of biological processes including disease pathogenesis, protein folding, receptor-ligand interactions, tumor-specific immune responses, etc [22]. Recently, increased evidences confirmed the importance of $\mathrm{N}$-glycosylation in molecular structures and biological networks in human cells. About $50 \%$ of human proteins, including both secreting and membrane proteins, can exhibit N-linked glycosylation patterns, which affect various aspects of biological behaviors of malignant tumor cells [23]. As altered glycosylation patterns are hallmarks of tumor progression, circulating tumor-specific glycoproteins are considered as candidate novel biomarkers in tumor diagnosis [24]. In small cell lung cancer (SCLC) study, sera from 54 SCLC patients and 29 health controls were screened by label-free proteomics and multiple reaction monitoring-mass spectrometry, four fucosylated proteins APCS, C9, SERPINA4 and PON1 were identified, APCS showed 87.5\% AUC and PON1 exhibited $91 \%$ AUC in extensive stage of SCLC [25]. Fucosylated glycoproteins carbohydrate antigen 19-1 and a-fetoprotein (AFP)-L3 could be used as tumor markers in sera from pancreatic and liver cancer patients [26]. However, the role of $\mathrm{N}$-glycosylation sites in early diagnosis of malignant tumor still needs extensive investigation.

In this study, we enrolled 40 clinical plasma samples, including 20 LUAD and 20 NL to screening tumor specific N-glycosylation sites. TMT-labeling and LC-MS/MS were conducted to investigate plasma protein profiles and identify differentiation proteins, as well as differential N-glycosylation sites between LUAD and NL. Our detection uncovered total 31 differential proteins and 39 differential N-glycosylation sites in LUAD samples, 17 were up-regulated and 22 were down-regulated. Extensive analysis revealed 39 sites harbored potential value in future clinical application. Among all candidate molecules, the most important site was ITGB3-680, which showed highest AUC (99.2\%), SN (95.0\%) and SP (95.0\%) in all proteins. Besides, APOB-1523 (AUC: 89.0\%), APOB-2982 (AUC: 86.8\%) and LPAL2-101 (AUC: 81.1\%) were also important in diagnosis of lung cancer. Due to limitation of single molecule in tumor diagnosis, we then conducted machine learning model to evaluate the combination of candidate $\mathrm{N}$-glycosylation sites. We divided all samples into training and testing groups and the result indicated that both groups harbored $100 \%$ AUC, which suggested that these $\mathrm{N}$-glycosylation sites could act as potential biomarkers in application of early diagnosis of LUAD.

In all proteins identified in our study, ITGB3 belonged to integrin family, which involved in tumor stress resistance and promoted metastatic progression of triple-negative breast cancer, and the study indicated that ITGB3 played a central role in endocytosis of extracellular vesicles by interacting and activating focal adhesion kinase (FAK) [27]. APOB was the main low-density lipoprotein and associated closely with poor prognosis in multiple malignant tumors, five independent single nucleotide polymorphism (SNPs) sites significantly associated with NSCLC survival in both discovery and replication datasets [28]. LPAL2 was lipoprotein (A) like 2, which was a pseudogene and contributed to tumor growth and metastasis, IncRNA 
of LPAL2 could be applied as biomarkers in malignant cholangiocytes [29]. CLU (clusterin) was involved in tumor progression by regulating C-Myc transcriptional repression and immune response, the microarray method and weighted expression profile revealed that decrease CLU could predict the poor survival of lung cancer [30]. ECM1 (extracellular matrix protein 1) was glycoprotein and promoted tumor progression by regulating variety of biological processes such as cell mineralization, proliferation, migration and angiogenesis, the study in gastric cancer revealed that ECM1 could enhance glucose metabolism by inducing FAK/SOX2 signaling pathway [31]. CD109 was a glycosyl phosphatidylinositol anchored protein and increased in multiple tumors including lung cancer, glioblastomas, melanomas and breast carcinoma [32]. CFH was a complement factor and high mRNA level related closely to progression of cutaneous squamous cell carcinoma [33]. In our study, N-glycosylation of these proteins were all important in diagnosis of lung adenocarcinoma.

However, our study also had several shortcomings. Firstly, this was a small cohort which contained only 40 participants ( 20 tumor patients and 20 normal controls). The second was that we only enrolled LUAD patients and NL controls. Thirdly, our study lacked of molecular mechanism of whether N-glycosylation sites could affect the biological functions of lung cancer. In our next study, we should contain benign lung diseases, including harmartoma, atypical hyperplasia, inflammatory pseudotumor, inflammatory nodules and infections, as well as lung squamous carcinoma (LUSC) and enlarged participants (at least 150 individuals in each group). Moreover, extensive research also needed to investigate the regulation mechanism of $\mathrm{N}$-glycosylation sites in lung cancer biological functions.

\section{Declarations}

- Ethics approval and consent to participate

This study was approved by ethics committee of China National Nuclear Corporation 416 Hospital

- Consent for publication

Not applicable

- Availability of data and materials

The datasets used and analyzed during the current study are available from the corresponding author on reasonable request.

- Competing interests

None declares

- Funding

None

Page 12/21 
- Author's contributions

ZQ designed the manuscript, KF performed data analysis and wrote the manuscript, QL and ZL collected samples and help to complete manuscript, ZL and CZ conceived of the study and participated in designation.

- Acknowledgements

We thanks medical record department for providing information of patients

\section{References}

1. Goldstraw P, Crowley J, Chansky K et al. The IASLC lung cancer staging project: proposals for the revision of the TNM stage groupings in the forthcoming (seven) edition of the TNM classification of malignant tumors. J Thorac. Oncol. 2007, 2: 706-714

2. Smith RA, Andrews KS, Brooks D, et al. Cancer screening in the United States, 2017: A review of current American Cancer Society guidelines and current issues in cancer screening. CA: a cancer journal for clinicians. 2017; 67: 100-21

3. Kinsinger LS, Anderson C, Kim J, et al. Implementation of Lung Cancer Screening in the Veterans Health Administration. JAMA internal medicine. 2017; 177: 399-406

4. Heschke $\mathrm{Cl}$, Yankelevitz DF, Libby DM et al. Survival of patients with stage I lung cancer detected on CT screening. N. Eng. J. Med. 2006, 355: 1763-1771

5. Alberle DR, Adams AM, Berg CD et al. Reduced lung-cancer mortality with low-dose computed tomographic screening. N. Eng. J. Med. 2011, 365: 395-409

6. Mulshine JL, D’Amico TA. Issues with implementing a high-quality lung cancer screening program. CA Cancer J. Clin. 2014, 64: 351-363

7. Patz JE, Campa MJ, Gottlin EB et al. Panel of serum biomarkers for the diagnosis of lung cancer. J Clin. Oncol., 2007, 25: 5578-5583

8. Grunnet M, Sorensen JB. Carcinoembryonic antigen (CEA) as tumor marker in lung cancer. Lung Cancer, 2012, 76: 138-143

9. Kosacka M, Jankowska R. Comparison of cytokeratin 19 expression in tumor tissue and serum CYFRA 21-1 levels in non-small cell lung cancer. Pol. Arch. Med. Wewn. 2009, 119: 33-37

10. Yihan Zhu, Yongfeng Yang, Yanwen Wang. Role of serum CA125 and CA199 concentration in diagnosis and prognosis evaluation of lung cancer patients. Int. J Clin. Exp. Pathol., 2016, 9: 53885396

11. Li Zhang, Dan Liu, Lei Li et al. The importance of circulating CYFRA21-1 in metastasis diagnosis and prognostic value compared with carcinoembryonic antigen and neuron-specific enolase in lung cancer patients. BMC Cancer. 2017, 17: 96 
12. Chu XY, Hou XB, Song WA et al. Diagnostic values of SCC, CEA, CYFRA21-1 and NSE for lung cancer in patients with suspicious pulmonary masses: a single center analysis. Cancer Biol. Ther., 2011, 11: 995-1000

13. Leighl NB, Page RD, Raymond VM, et al. Clinical Utility of Comprehensive Cell-free DNA Analysis to Identify Genomic Biomarkers in Patients with Newly Diagnosed Metastatic Non-small Cell Lung Cancer. Clinical cancer research, 2019; 25: 4691-700.

14. Van der Pol E, Boing AN, Hamison $P$ et al. Classification, function, and clinical relevance of extracellular vesicles. Pharmacol. Rev. 2012, 64: 676-705

15. Marquette $\mathrm{C}-\mathrm{H}$, Boutros J, Benzaquen J, et al. Circulating tumour cells as a potential biomarker for lung cancer screening: a prospective cohort study. Lancet Respiratory medicine. 2020; 8: 709-16.

16. Tan HT, Low J, Lim SG, et al. Serum autoantibodies as biomarkers for early cancer detection. The FEBS journal. 2009; 276: 6880-904

17. Thery C, Zitvogel L, Amigorena S. Exosomes: composition, biogenesis and function. Nat. Rev. Immunol. 2002, 2: 569-579

18. Li AC, Zhang TB, Zheng M et al. Exosomal proteins as potential markers of tumor diagnosis. J. Hematol. Oncol. 2017, 10: 175

19. Li WH, Li CY, Zhou T et al. Role of exosomal proteins in cancer diagnosis. Molecular Cancer. 2017, 16: 145

20. Lacombe J, Mangé A, Jarlier M, et al. Identification and validation of new autoantibodies for the diagnosis of DCIS and node negative early-stage breast cancers. International journal of cancer. 2013; 132: 1105-13

21. Heo SH, Lee SJ, Ryoo HM et al. Identification of putative serum glycoprotein biomarkers for human lung adenocarcinoma by multilectin affinity chromatography and LC-MS/MS. Proteomics, 2007, 7: 4292-4302

22. Wang CQ, Gao WJ, Yan S et al. N-glycome and N-glycoproteome of a hematophagous parasitic nematode Haemonchus. Comput. Struct. Biotech. J., 2021; 19: 2486-2496

23. Miyoshi E, Moriwaki K, Nakagawa T. Biological function of fucosylation in cancer biology. J Biochem., 2008, 143: 725-729

24. Kobata A, Amano J. Altered glycosylation of proteins produced by malignant cells, and application for the diagnosis and immunotherapy of tumors. Immunol. Cell. Biol., 2005, 83: 429-439

25. Ahn JM, Sung HJ, Yoon YH et al. Integrated glycoproteomics demonstrates fucosylated serum paraoxonase 1 alterations in small cell lung cancer. Mol. Cell. Proteom., 2014, 13: 30-48

26. Aoyagi Y, Saitoh A, Suzuki Y et al. Fucosylation index of a-fetoprotein, a possible aid in the early recognition of hepatocellular carcinoma in patients with cirrhosis. Hepatology, 1993, 17: 50-52

27. Fuentes P, Sese M, Guijarro PJ et al. ITGB3-mediated uptake of small extracellular vesicles facilitates intercellular communication in breast cancer cell. Nat. Commun., 2020, 11: 4261 
28. Deng W, Liu HL, Luo S et al. APOB genotypes and CDH13 haplotypes in the cholesterol-related pathway genes predict non-small cell lung cancer survival. Cancer Epide. Bio. Prev., 2020, 9: 12041213

29. Han BW, Ye H, Wei PP et al. Global identification and characterization of IncRNAs that control inflammation in malignant cholangiocytes. BMC Genomics, 2018, 19: 735

30. Wu X, Wang LL, Feng $F$ et al. Weighted gene expression profiles identify diagnostic and prognostic genes for lung adenocarcinoma and squamous cell carcinoma. J Int. Med. Res., 2019, 48: 1-12

31. Gan L, Meng J, Xu M et al. Extracellular matrix protein 1 promotes cell metastasis and glucose metabolism by inducing integrin beta4/FAK/SOX2/HIF-1a signaling pathway in gastric cancer. Oncogene, 2018, 37: 744-755

32. Lee KY, Shueng PW, Chou CM et al. Elevation of CD109 promotes metastasis and drug resistance in lung cancer via activation of EGFR-AKT-mTOR signaling. Cancer Science, 2020, 111: 1652-1662

33. Riihila PM, Nissinen LM, Ala-aho R et al. Complement Factor $\mathrm{H}$ : a biomarker for progression of cutaneous squamous cell carcinoma. J Invest. Dermat., 2014, 134: 498-506

\section{Figures}

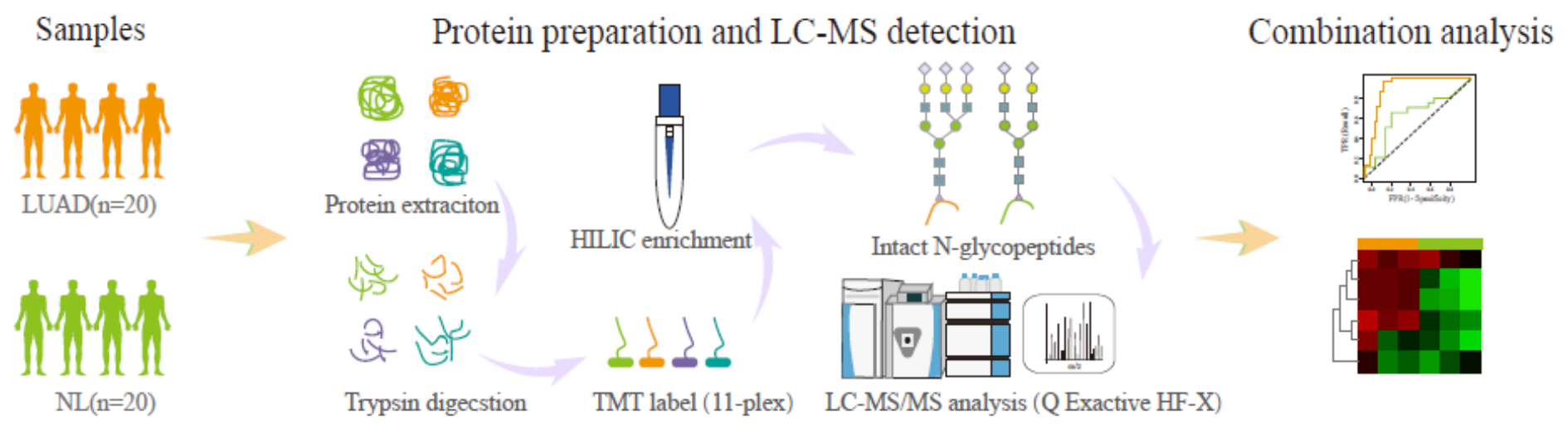

\section{Figure 1}

Workflow of N-glycosylation analysis in LUAD patients and NL controls. Trypsin was added into all samples for protein digestion and then processed by TMT kit/iTRAQ kit. High pH reverse-phase HPLC was performed to fractionate tryptic peptides and then dissolved in NETN buffer for enrichment. The peptides were then subjected to tandem mass spectrometry (LC-MS/MS) in Q ExactiveTM Plus. A datadependent procedure was then conducted to peptides and alternated between one MS scan followed by 20 LC-MS/MS scans with 15.0 s dynamic exclusion. 

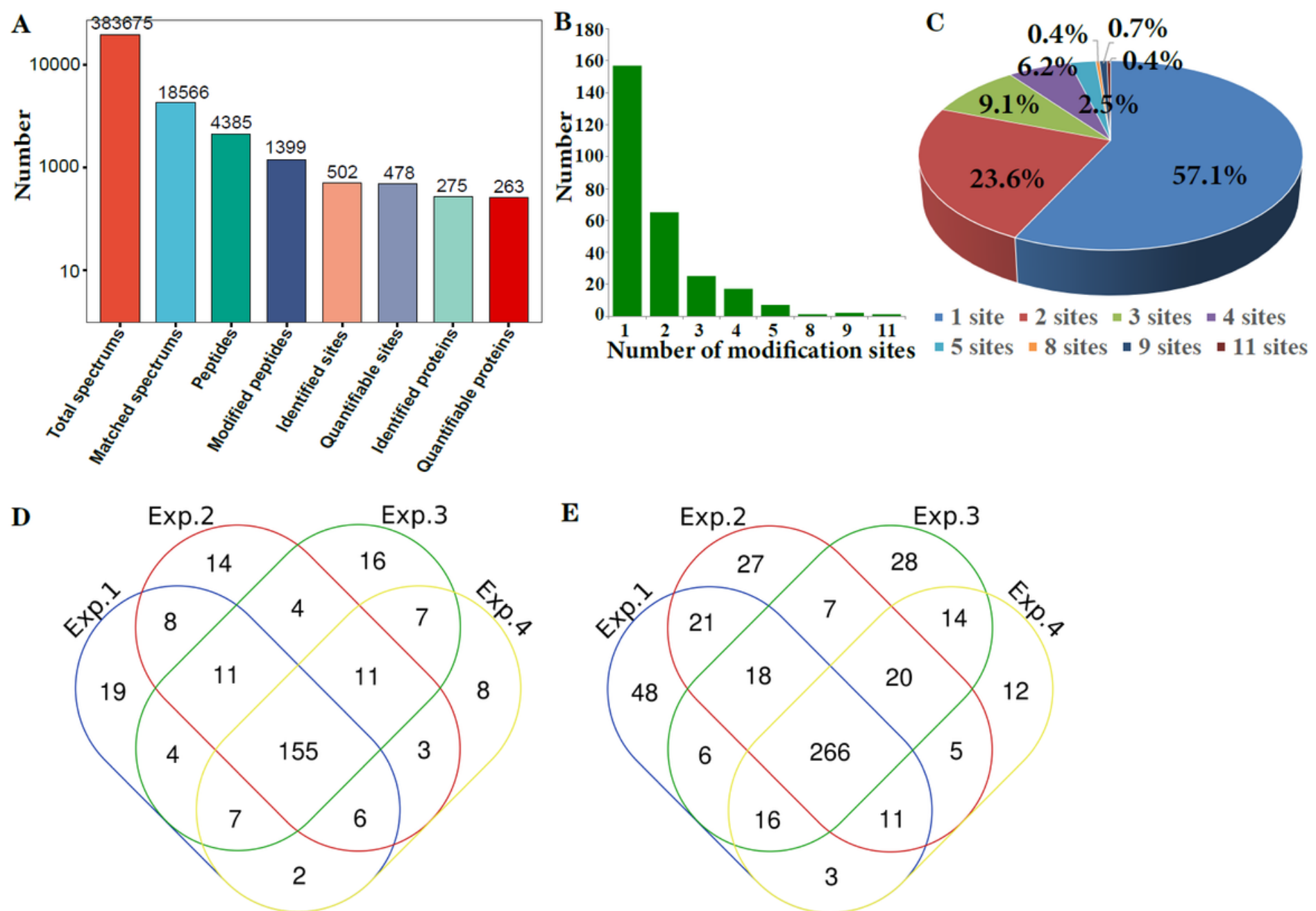

\section{Figure 2}

Characteristics of identified N-glycoproteins.
A. Total number of identified $\mathrm{N}$-glycosylation proteins.
B. The number of modification sites per protein.
C. Pie chart showed the number and proportions of single and multiple N-glycosylated sites in LUAD.
D. Overlap numbers of glycoproteins in LUAD samples.
E. Overlap numbers of glycosylation sites in LUAD samples 


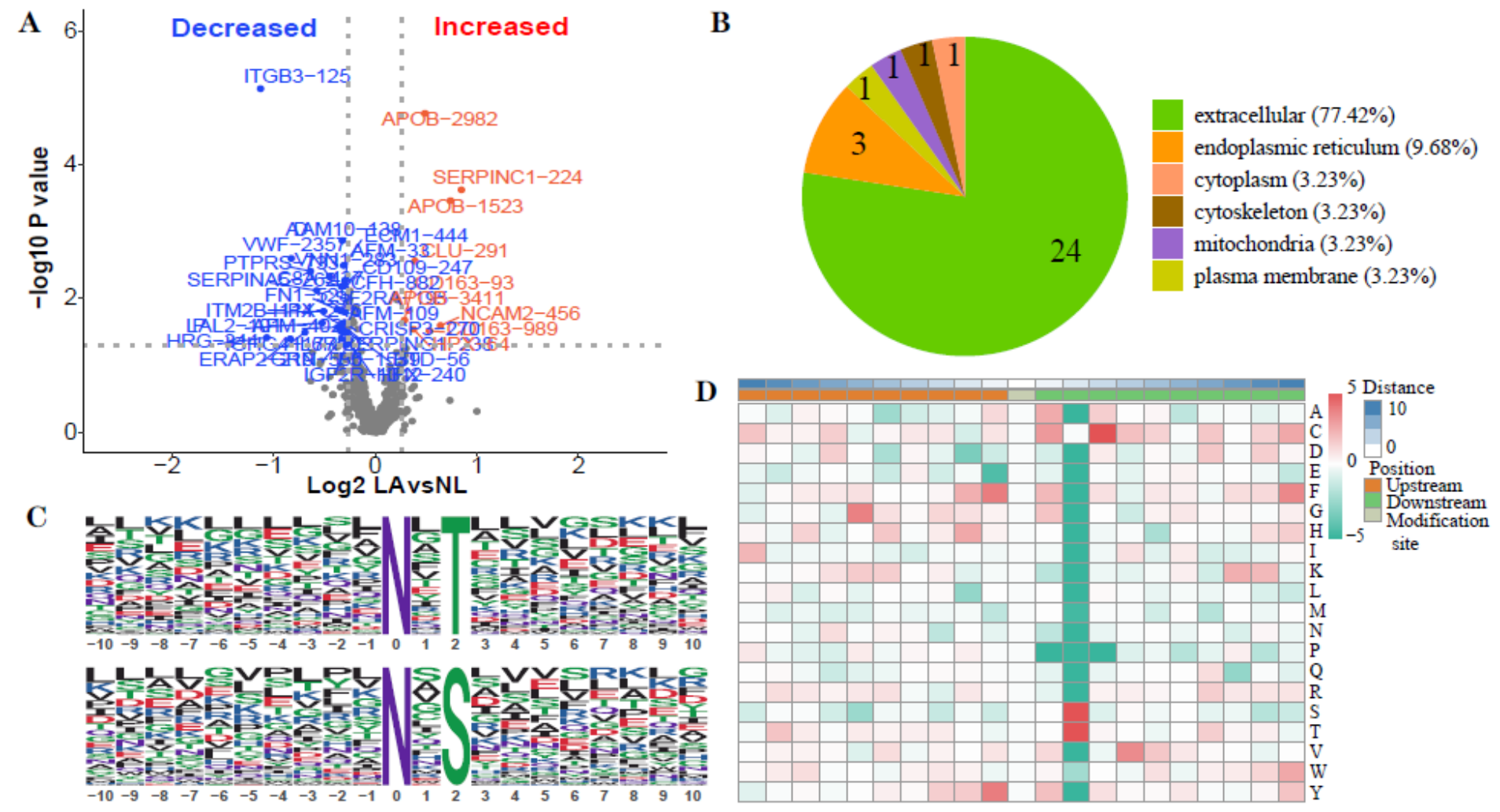

\section{Figure 3}

Disease-associated variations in N-glycopeptide abundance in LAUD.

A. Volcano revealed increased and decreased N-glycosylation sites in LUAD and NL samples.

B. Classification of identified N-glycosylation proteins based on subcellular loction.

C. Sequence motifs located nearby the target asparagine in enriched glycosylation sites.

D. Heatmap showing the relative frequency of amino acids in the proximity of asparaine (enrichment, red; depletion, green) 
A Biological Process

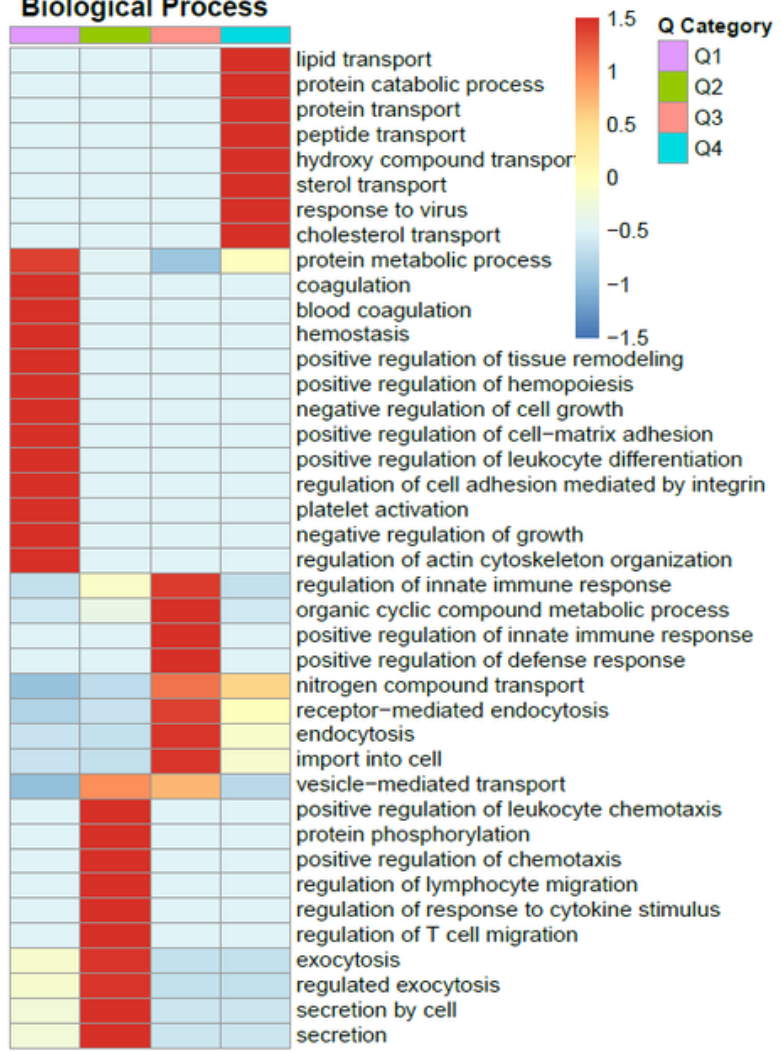

\section{Molecular Function}

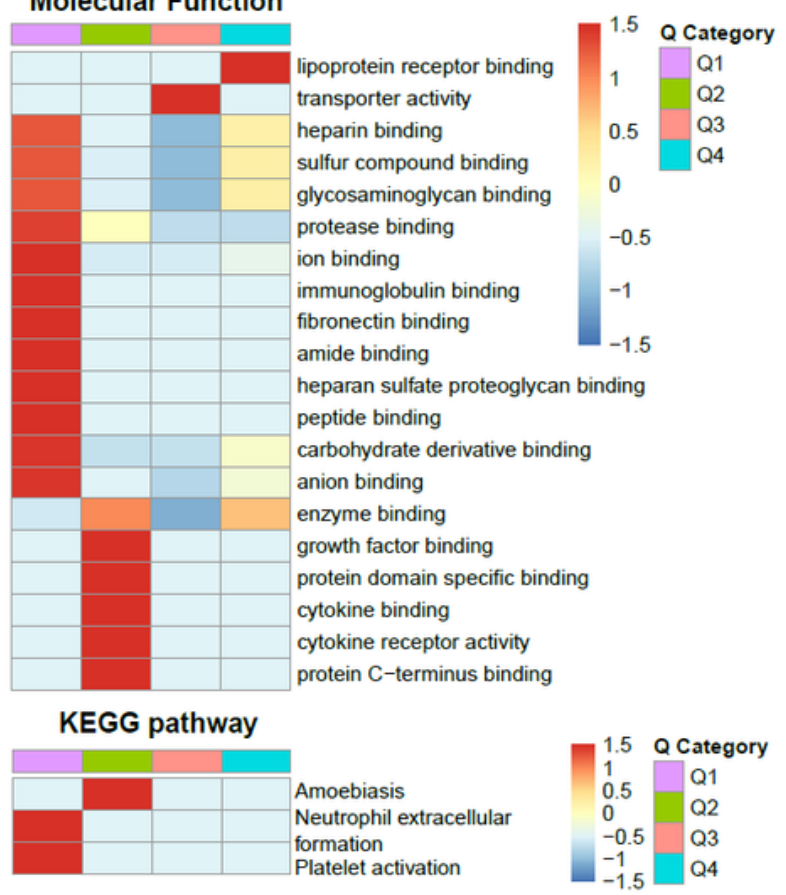

B Cellular Component

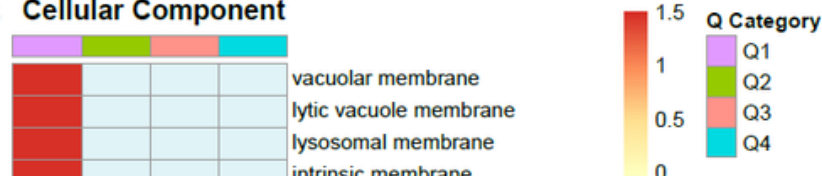
lysosomal membrane intrinsic membrane integral membrane vesicle membrane cytoplasmic vesicle membrane $\quad-1$ whole membrane bounding membrane of organelle -1.5 organelle membrane platelet alpha granule intracellular vesicle cytoplasmic vesicle intracellular organelle lumen organelle lumen membrane-enclosed lumen cytoplasmic vesicle lumen vesicle lumen secretory granule lumen trans-Golgi network Golgi-associated vesicle transport vesicle secretory granule secretory vesicle endoplasmic reticulum lumen protein-lipid complex plasma lipoprotein particle lipoprotein particle endocytic vesicle membrane endocytic vesicle endocytic vesicle lumen

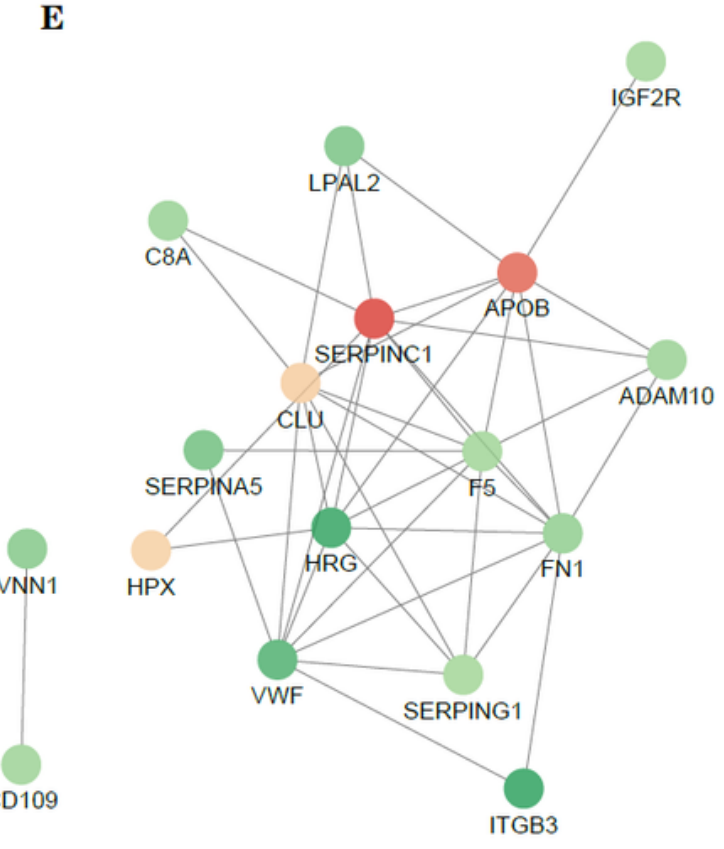

Figure 4

Annotation of differential N-glycosylated proteins in LUAD samples.

A-D. Functional enrichment-based clustering analysis for quantified glycoproteome. A: biological process analysis, B: cellular component, C: molecular function analysis, D: KEGG pathway analysis. 
E: PPI network analysis. Each node represents an $\mathrm{N}$-glycoprotein and each edge represents the interaction between proteins.
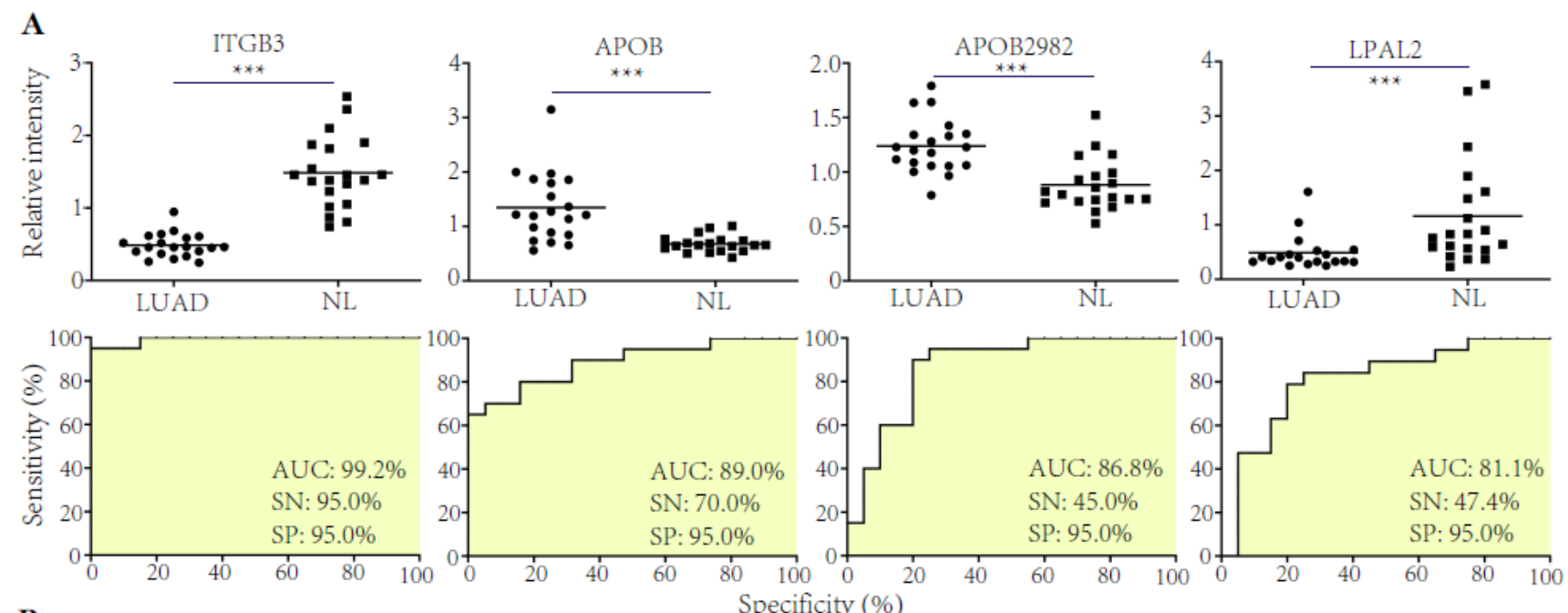

B
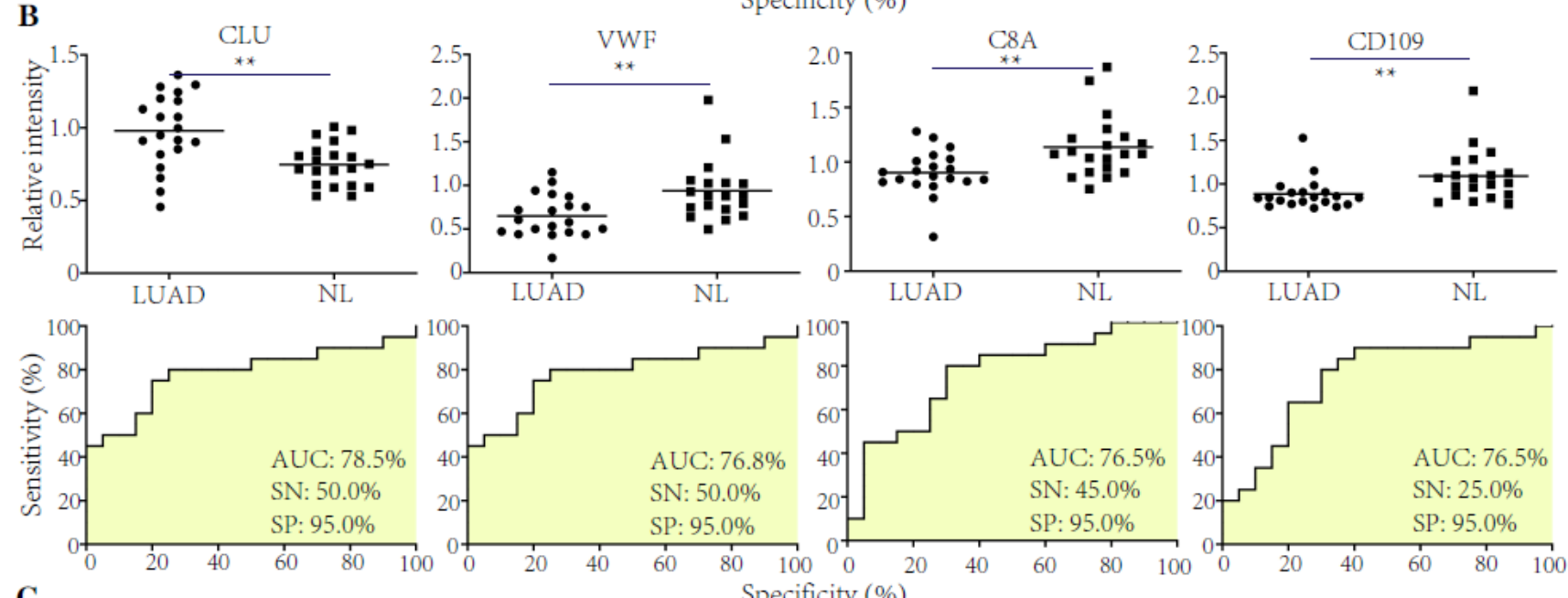

C
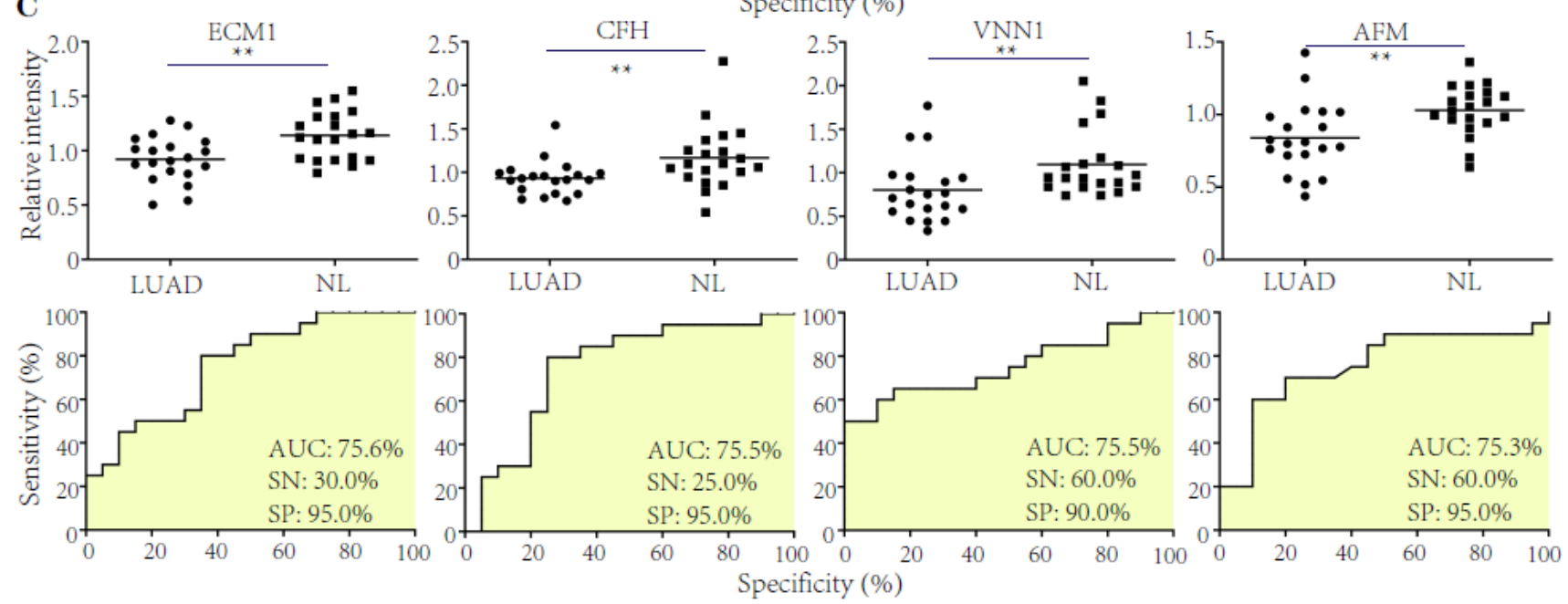

\section{Figure 5}

The concentration and ROC analysis of differential N-glycosylation sites identified in LUAD patients. 
A. The concentration and ROC analysis of $\mathrm{N}$-glycosylation sites with AUC>80.0\%.

B-C. The concentration and ROC analysis of $\mathrm{N}-$ glycosylation sites with $\mathrm{AUC}>70.0 \%$.

${ }^{\star \star} p<0.01, * \star \star p<0.001$

A

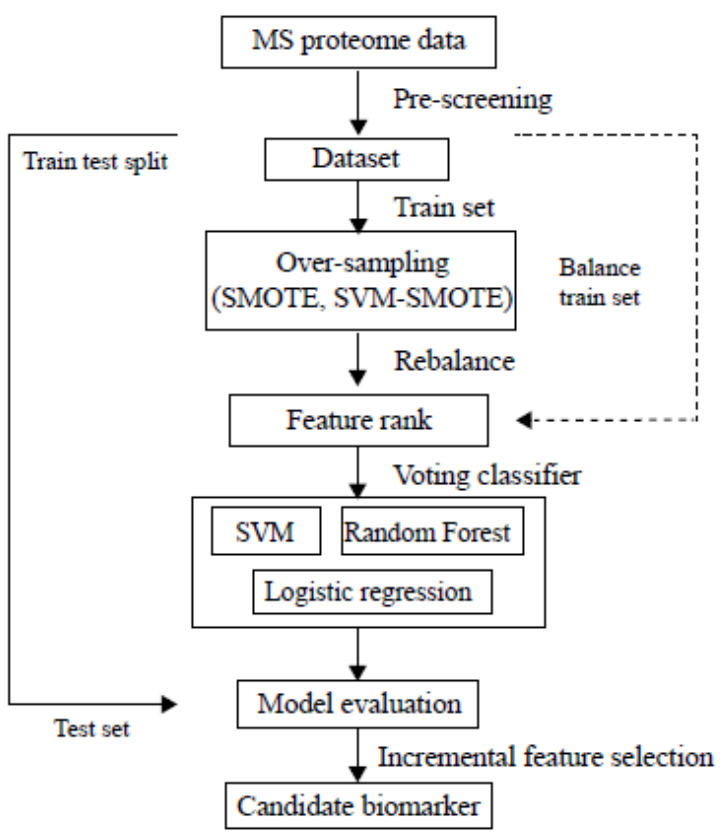

C

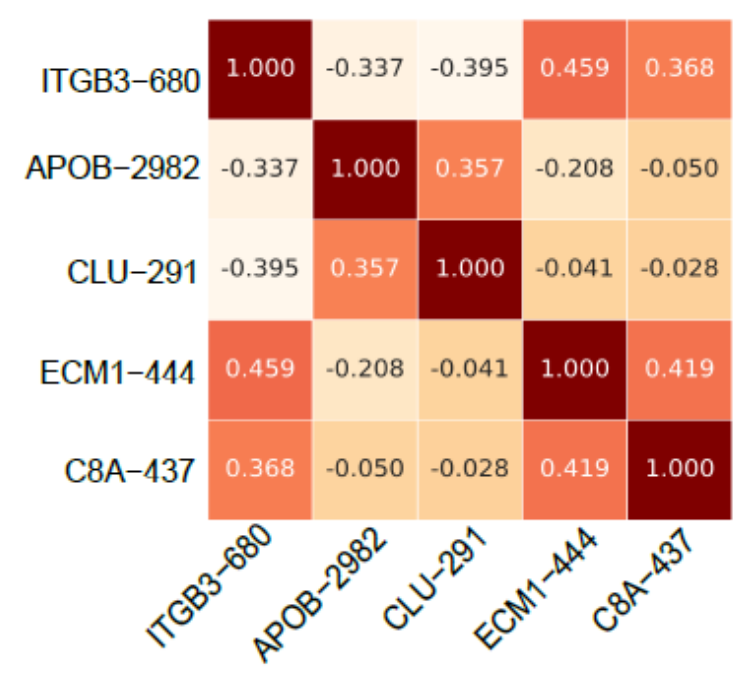

B

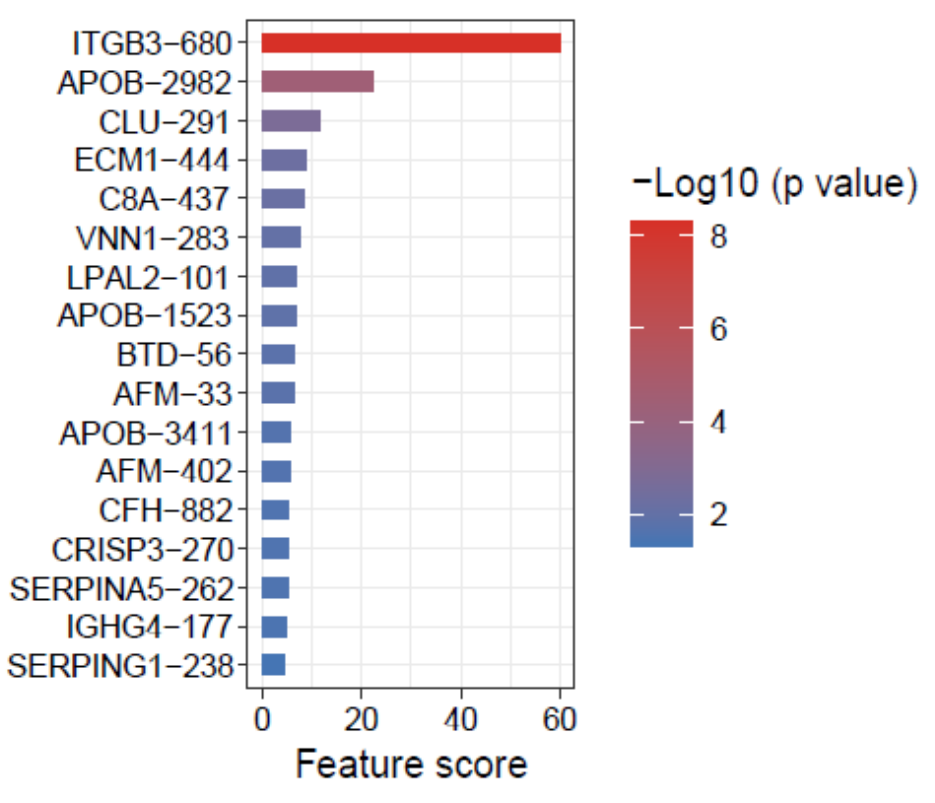

D

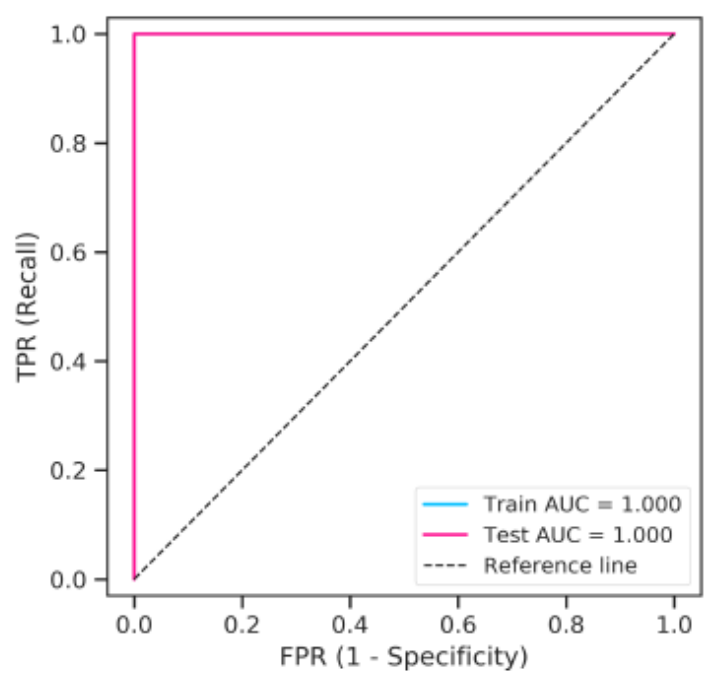

Figure 6

Combination analysis of candidate biomarkers by machine learning.
A. Analysis schema of machine learning.
B. Feature score of candidate N-glycosylation sites identified by feature selection.
C. Pearson correlation coefficient in Top $5 \mathrm{~N}$-glycosylation sites in feature score. 
D. AUC in training (16 cases) and test set ( 4 cases) by plotting the true positive rate against the false positive rate under different cut-off values 